\title{
Application of Proteus Simulation in Electronic and Information Engineering Specialty
}

\author{
Hui Li, Ming Zhao, Yuru Zhang, Xiaodong Su, Haitao Jiang \\ School of Computer and Information Engineering, Harbin University of Commerce, Harbin, China \\ hrbcu_lh@163.com
}

Keywords: Electronic and Information Engineering; Practice Teaching; Proteus

\begin{abstract}
This article researches the application of Proteus simulation in Electronic and Information Engineering specialty. The relevant practice teaching of the course has been cascaded, can not only help students to link up theory knowledge, and also inspire further the enthusiasm and motivation of scholars. Based on Proteus, this paper introduces some cases to connect the practical teaching of several related courses of electronic information engineering. This teaching method has been proved that it not only ensures the continuity of knowledge, but also no affection in the course independence and expansibility. Above all, it improves students' interest in learning and exploration motivation.
\end{abstract}

\section{Introduction}

Electronic and information engineering is to train students to possess the ability of electronic product design, research and development, signal and information processing, and information system integration. Nowadays, with the rapid development of science and technology, electronic and information technology develops rapidly and new products emerge endlessly. In order to improve the innovation spirit and entrepreneurial ability, Protues simulation must be carried out, and the important step is practical teaching reform [1].

\section{Sort Out the Professional Curriculum Structure}

There are three modules of the electronic and information engineering core courses:

\subsection{Basic course module}

Basic course modules mainly include the common courses such as "Circuit Analysis", "Analog Electronics Technology", and "Digital Logic", which are the basis for learning subsequent courses.

\subsection{Information and communication engineering course module}

Information and communication engineering course module mainly includes "Signals and Systems", "Communication Clectronic Circuit", "Random Signal Processing”, “Information Theory and Coding”, "Digital Signal Processing” and "Digital Image Pocessing”.

\subsection{Control science and engineering course module}

Control science and engineering course module mainly includes "Application of Single-Chip Microcomputer”, "Sensor Technology”, "Embedded Systems”, "Wireless Sensor Network” and “Automatic Control Theory” [2].

Through sorting out the curriculum structure, we can research on the relevant specialized courses by point to line practice teaching mode. Proteus software has been introduced in the process of practice teaching, and dynamic simulation teaching has been improved in "Circuit Analysis", “Analog Electronics Technology”, and "Digital Logic” [3]. 


\section{Rebuild the core competence of electronic and information engineering specialty}

The problem of construction of new engineering is how to change from the instrumental education focusing on tools and languages to engineering education. Students are trained to become cross-system, cross-integrated, engineering methodological technical talents. To build a solid professional foundation is the first step. Many problems are difficult to break through for some graduates and in complex engineering environment after working some time. Professional basis is the foundation of personal growth. The second step is splicing crossover. "Cloud", in many enterprises, is a replacement of the original computer room. It is the normal capability of electronic and information engineering specialty. However, "Mobile Internet", whether as a carrier of APP or some small programs, has been developed with many frameworks. So it is also the normal capability of electronic and information engineering specialty.

Based on the core competence of computer system capability has been established, the cross fusion of Big Data and AI has been the new core. On the basis of solid ability of computer system, the teaching content and practice case would be keep synchronized with enterprise and industry. At last, the core of computer professionals ability and competitiveness will be formed.

Fig. 1 shows the hierarchy of computer system ability training.

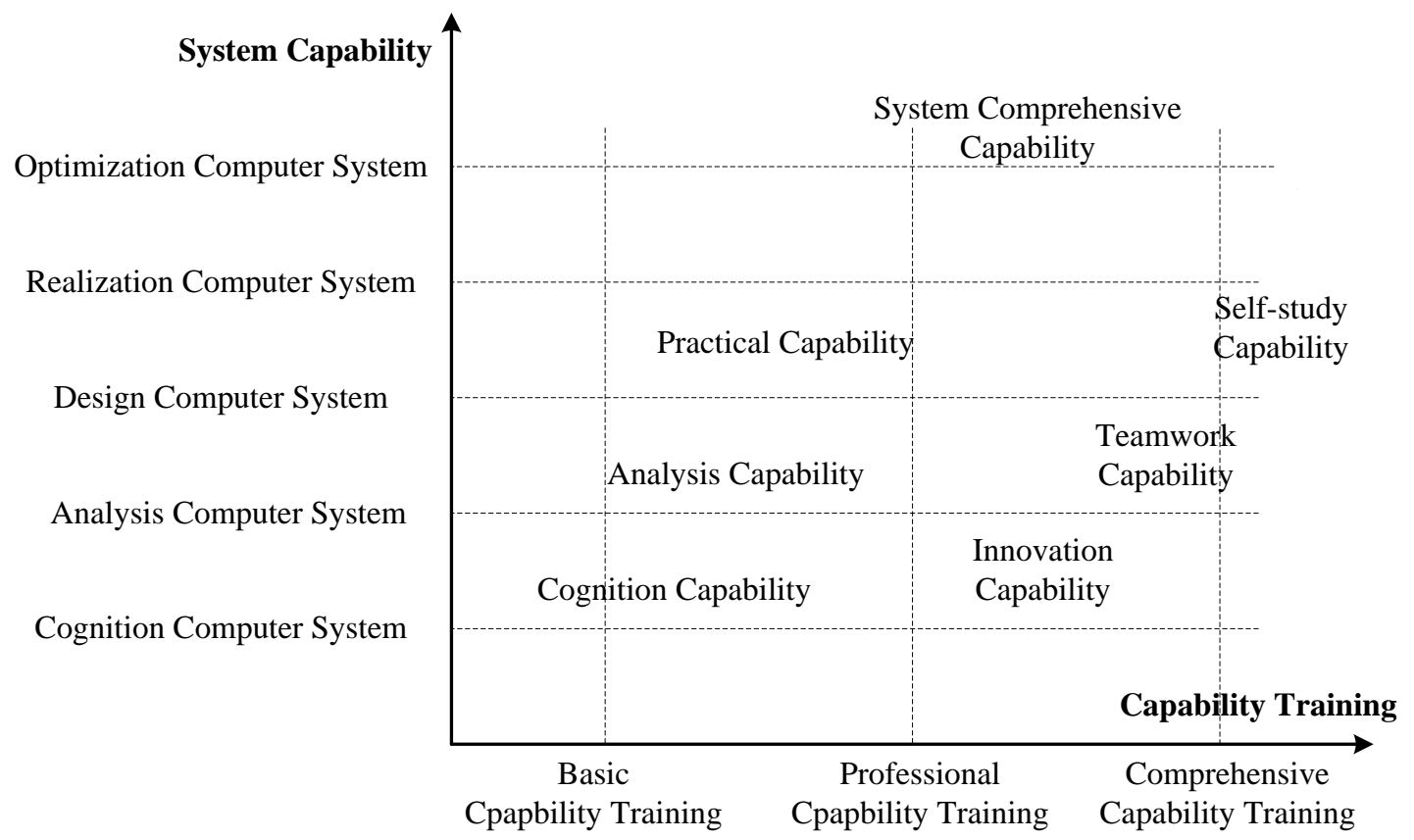

Figure.1 Hierarchy of computer system ability training.

\section{Application of Proteus Simulation in Electronic and Information Engineering Specialty}

\subsection{Proteus simulation case based on waveform generator in "Circuit Analysis"}
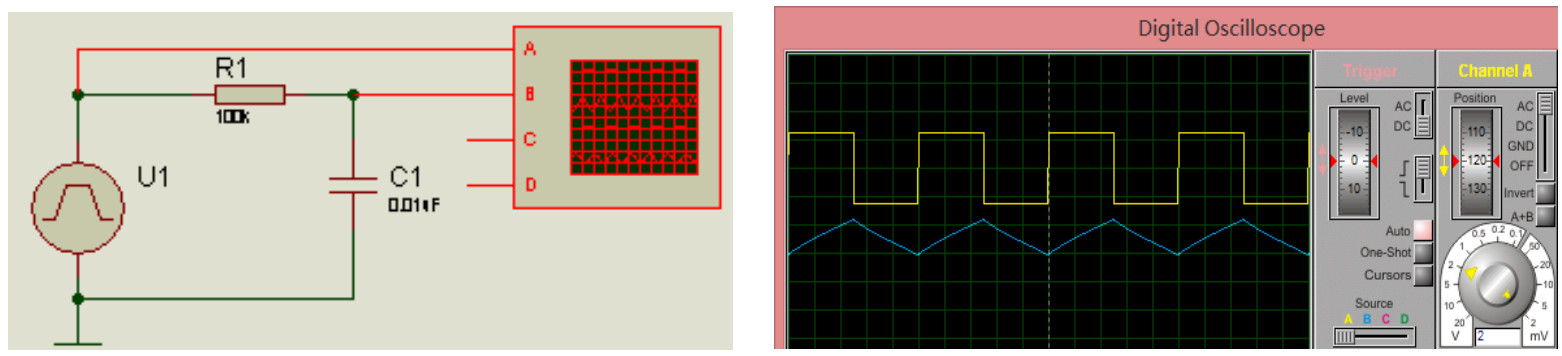

Figure.2 Integrated circuit and waveform figure

Fig. 2 is the simulation circuit diagram of square waves convertes into triangular waves with 
integrated circuit in "Circuit Analysis", and square wave convertes to positive and negative pulse waveform with integrated differential circuit have shown in Fig.3.
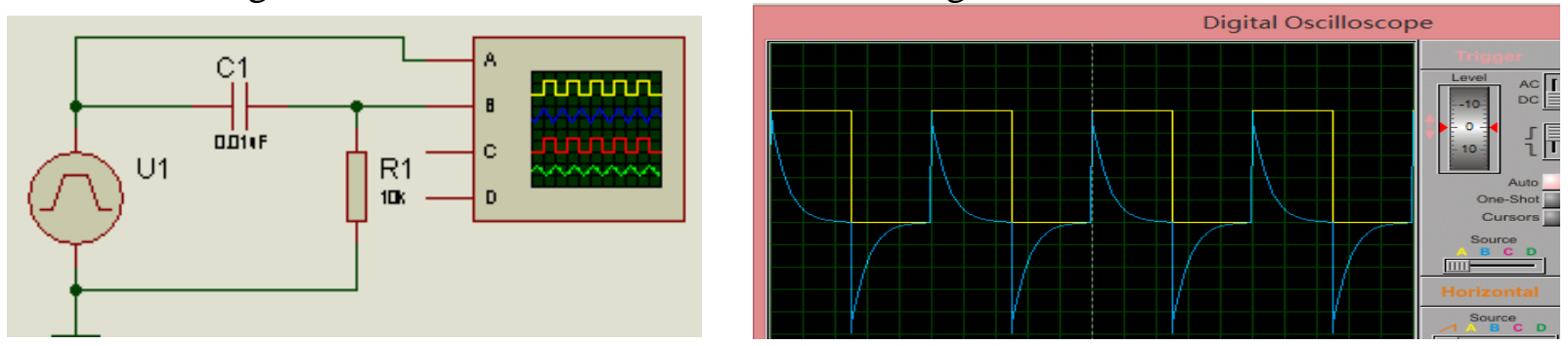

Figure.3 Differential circuit and waveform figure

\subsection{Proteus simulation case based on $\mu \mathrm{A} 741$ in "Analog Electronics Technology"}

In "Analog Electronics Technology" course, waveform generator experiment based on the integrated operational amplifier has been set, and sine wave, square wave and triangular wave would been produced by $\mu$ A741. Fig. 4 is the waveform figure of waveform generator based on $\mu$ A741 [4].
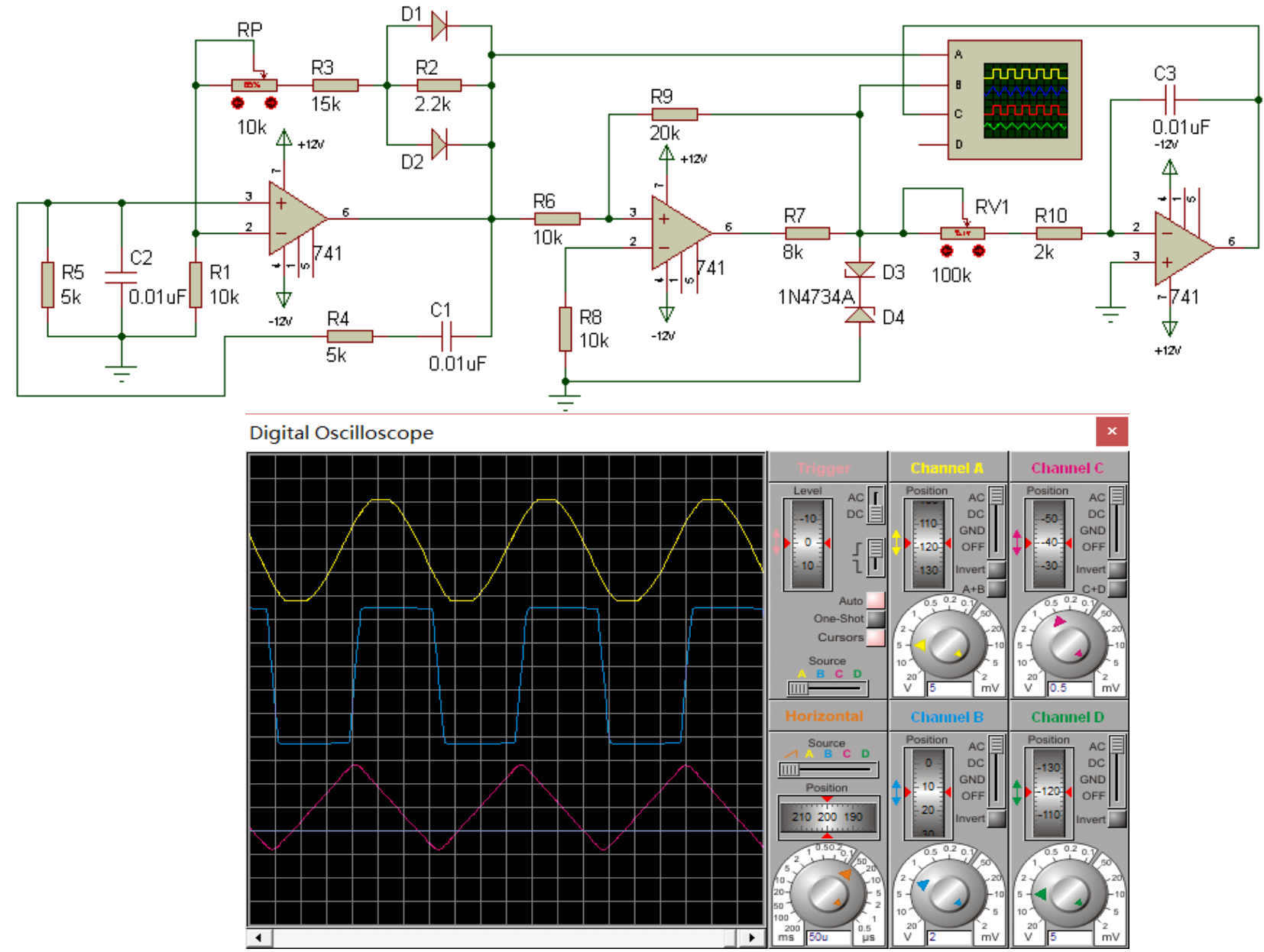

Figure.4 Circuit and waveform figure of waveform generator based on $\mu \mathrm{A} 741$

\subsection{Protues simulation case based on ADC0808 for "Digital Logic"}

The output waveform of the unipolar saw tooth wave circuit is shown in Fig 5.

There is a 256 system adding counter which consisted with two pieces of 74161 [5], and the output value is $00000000 \sim 111111111111$. At last, the output results are displayed by oscilloscope. We can change the waveform period by CP pulse frequency. As shown in Fig.6, the output voltage signal of operational amplifier uA741 is unipolar and the voltage range is $0-5 \mathrm{~V}$. 

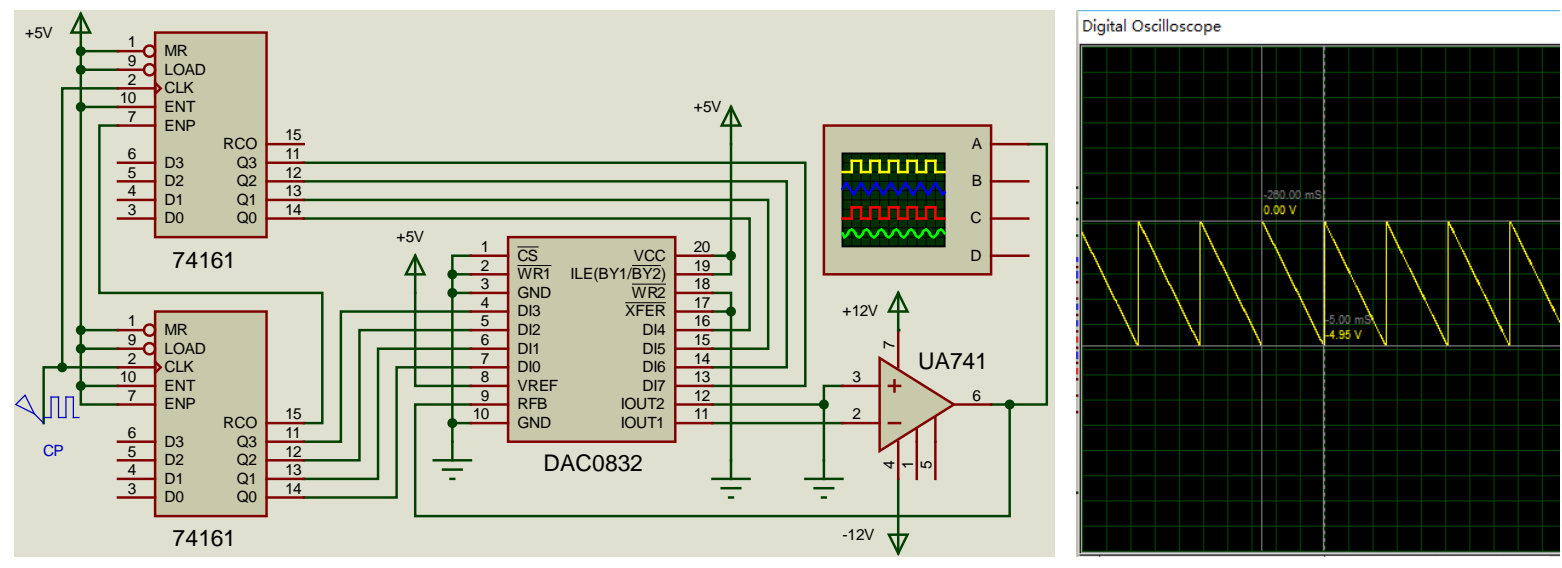

Figure.5 Output waveform of the unipolar sawtooth wave circuit
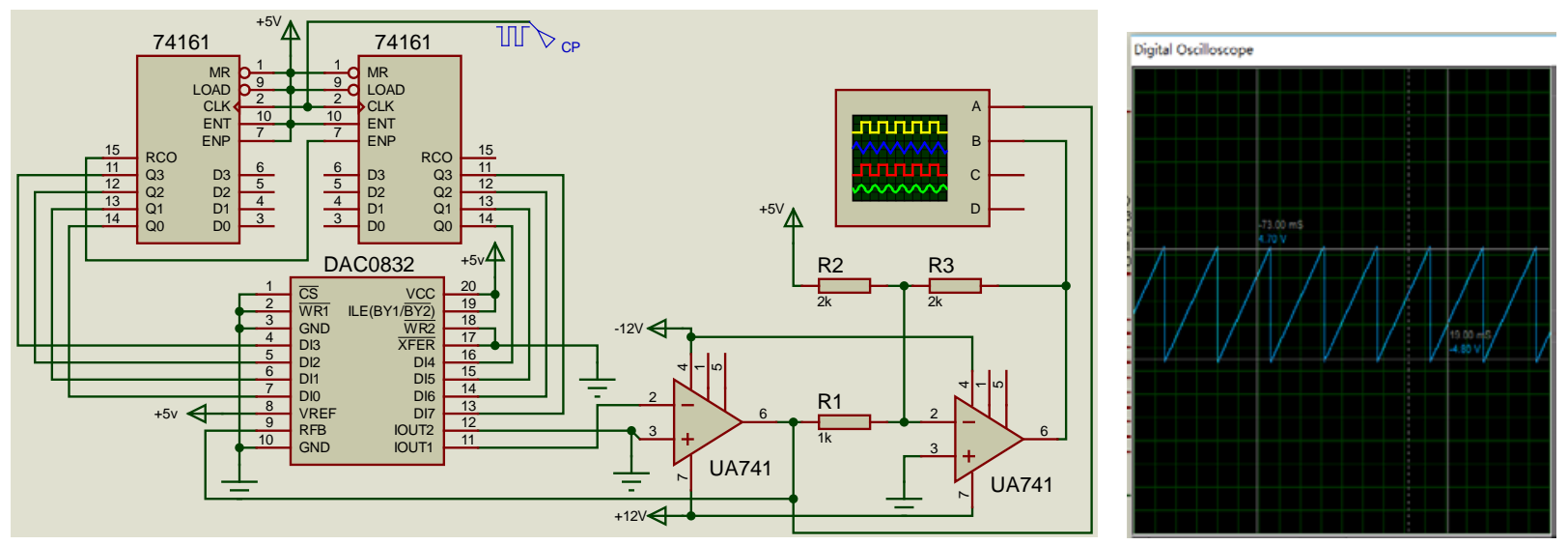

Figure.6 Output waveform of the bipolar sawtooth wave circuit

\section{Summary}

According to the idea of point to line practice teaching in this paper, we can also deeply study the training scheme and teaching outline of relevant specialized courses, and find the common points of relevant courses. It enables students to combine professional learning with scientific exploration and employment ability and make contributions to the society.

\section{Acknowledgements}

This work was financially supported by Cooperative and Cooperative Education Project of Ministry of Education of the People's Republic of China (201601023031, 201702029029, 201702064010), Teaching Reform Research Projects of Heilongjiang Education Department (SJGY20170055, SJGY20170075), Teaching Reform Research Projects of Harbin University of Commerce (SJXM2018A004, HSDJY014), Natural Science Foundation of Heilongjiang Province of China (F2015045), University Nursing Program for Young Scholars with Creative Talents in Heilongjiang Province (UNPYSCT-2016063) and Science Foundation of Harbin Commerce University (17XN060).

\section{References}

[1] Dai Fen, Wang Weixing, i Zhen, Sun Daozong, Deng Xiaoling, Jiang Sheng. Research on the Cultivation of Undergraduate Talents in Electronic and Information Engineering Specialty, China Electronics Education No.1 (2016)

[2] Yan Gaizhen, Xu Chaoshen, Li Shuangxi. Exploration on the Engineering Education Oriented 
Electronic Information Specialty Construction. academic research.

[3] LIU Chun-ling, HE Ting, LU Hai-tian, WANG Chun-wu. Construction and practice of the course groups with different orientations in electronic information engineering major. Journal of Science of Teachers College and University (2018)

[4] Quansheng Yang etal. Construction of a computer system practice platform that combines theory with practice. Computer education. Vol.33 No.1 (2018)

[5] Yuru Zhang, etal. Digital logic circuit design (2 ${ }^{\text {nd }}$ edition). Harbin: Harbin Institute of Technology Press, (2018). 